\title{
Generalized Synchronization of Stochastic Discrete Chaotic System with Poisson Distribution Coefficient
}

\author{
Shao-juan Ma, Duan Dong, and Jie Zheng \\ School of Information and Computation Science, Beifang University of Nationalities, Yinchuan 750021, China \\ Correspondence should be addressed to Shao-juan Ma; sjma.math@yahoo.com
}

Received 29 March 2013; Revised 10 June 2013; Accepted 1 July 2013

Academic Editor: Gualberto Solís-Perales

Copyright ( 2013 Shao-juan Ma et al. This is an open access article distributed under the Creative Commons Attribution License, which permits unrestricted use, distribution, and reproduction in any medium, provided the original work is properly cited.

\begin{abstract}
This paper addresses the generalized synchronization of stochastic discrete chaotic systems with Poisson distribution coefficient. Firstly, based on the orthogonal polynomial approximation theory of discrete random function in Hilbert spaces, the discrete chaotic system with random parameter is transformed into its equivalent deterministic system. Secondly, a general method for the generalized synchronization of discrete chaotic system with random parameter is presented by Lyapunov stability theory and contraction theorem. Finally, two synchronization examples numerically illustrated that the proposed control scheme is effective for any stochastic discrete system.
\end{abstract}

\section{Introduction}

Synchronization is a kind of typical collective behavior and basic motion in nature. Since Pecora and Carroll [1] showed that it is possible to synchronize the coupled chaotic dynamical system with different initial conditions, chaos synchronization has been extensively studied due to its theoretical challenge and great potential application in secure communication, neuroscience, encoding message, chemical reaction, and complex networks [2]. Therefore, many different types of synchronization methods such as phase (antiphase) synchronization [3], partial synchronization [4], projective synchronization [5, 6], lag synchronization [7], complete synchronization [8], Q-S synchronization [9], fast synchronization [10], and adaptive impulsive synchronization [11] have been presented in continuous-time chaotic systems in the past two decades.

But from the applied point of perspective, many systems in daily life can be illustrated by the discrete-time system, and that system compared with continuous system is more suitable for simulation by computer. At the same time, some continuous-time systems are based on discrete-time system as the numerical approximation method, for instance, the Runge-Kutta method and the predictor-corrector method [12]. In order to analyze the science computation and numerical simulation accurately in the field of engineering, many researchers have gradually begun to think about the performance of dynamical system in practice; for example, communication signals which are transferred one by one in an interval and the number of butterflies which is a seasonal cycle time are usually described as the discretetime system. Besides, many mathematical models related to nonlinear phenomena are defined as discrete-time dynamical system in biological process, population growth, and neural networks $[13,14]$. Nowadays, a growing number of scholars start to concentrate on the numerical methods which could exactly reflect the dynamic behavior about the origin systems. Therefore, study of discrete-time system is of great value in theory and practical application. Recently, the research of synchronization about discrete-time system has made some progress. Zhang and Liu [15] have investigated impulsive chaotic synchronization of discrete-time-switched systems with state-dependent switching strategy. Taking advantage of contraction mapping theorem, adaptive function projective synchronization of discrete chaotic systems with unknown parameters is considered by $\mathrm{Wu}$ and $\mathrm{Fu}$ [16]. HengsterMovric et al. [17] have proposed a new method for synchronization based on the relation of graph eigenvalues to a bounded circular region in the complex plane that depends on the agent dynamics and the Riccati solution. In [18], the 
controlled synchronization problem for a class of nonlinear discrete-time chaotic systems subjected to limited communication capacity has been introduced by Liu et al. A fuzzy model-based adaptive approach to synchronize two different discrete-time chaotic systems with unknown parameters is presented by Vasegh and Majd [19]. Su and Ding [20] have used the Euler method to a discrete delayed chaotic system and achieved the globally exponential synchronization under the negative feedback control. There have already been a lot of research results about synchronization of the discrete-time chaotic systems [21-24].

It is worth pointing out that these stochastic factors like uncertainty of the structure parameter, perturbation of external noise, and stochastic input are ubiquitous in nature, society, economy, and realistic engineering. Under normal circumstances, those stochastic factors just play a minor influence. However, when the development of the system needs to make a choice, it will become a dominant factor which could affect the trend of deterministic system. As we investigate the actual population growth and biological process, the incomplete observations such as natural disasters, weather changes, and technology factors are unavoidably arousing uncertainties of mathematical models; especially, phenomena which appear in stochastic system at critical value due to the effects of random physical parameters are always unforeseen in the deterministic system and interfere seriously with daily production and life. Therefore, the system with random parameter can properly represent the actual mathematical model, and its research will possess more practical significance.

Motivated by the previous discussion, we have found that the research about synchronization of stochastic discrete chaotic system is little. Based on this, in this paper, considering the influence of stochastic factor on system parameter and through statistical characteristic of random variable, we build a kind of stochastic discrete chaotic system with random parameter and propose active control method to achieve the generalized synchronization of stochastic discrete system with random parameter. This paper is organized as follows. The orthogonal polynomial approximation of the stochastic discrete chaotic system with random parameter is given in Section 2. Section 3 proposes the generalized synchronization of discrete chaotic systems with random parameter under an active controller. Two examples are given to demonstrate the effectiveness of proposed method in Section 4. Finally, conclusions are drawn in Section 5.

\section{Orthogonal Polynomial Approximation of Stochastic Discrete System with Poisson Distribution Coefficient}

In this paper, we will take stochastic Lorenz discrete system and stochastic Henon map as examples.

The stochastic Lorenz discrete system is described as

$$
\begin{gathered}
x(n+1)=(1+\alpha \beta) x(n)-\beta x(n) y(n), \\
y(n+1)=(1-\beta) y(n)+\beta x^{2}(n),
\end{gathered}
$$

where $\beta$ is a deterministic parameter, and $\alpha$ is a random parameter which can be expressed as

$$
\alpha=\bar{\alpha}+\delta k
$$

where $k$ is a random variable which obeys density function of the Poisson distribution $p_{k}$ with standard deviation $\lambda$, and $\delta$ is the strength of random disturbance.

So, the response of the system (1) with random parameter can be approximately expressed by the following series under condition of the convergence in mean square:

$$
\begin{aligned}
& x(n, k)=\sum_{i=0}^{M} x_{i}(n) P_{i}(k), \\
& y(n, k)=\sum_{i=0}^{M} y_{i}(n) P_{i}(k),
\end{aligned}
$$

where $x_{i}(n)=\sum_{k=0}^{N} p_{k} x(n, k) P_{i}(k), y_{i}(n)=\sum_{k=0}^{N} p_{k} y(n, k)$ $P_{i}(k), P_{i}(k)$ is the $i$ th Charlier orthogonal polynomial, and $M$ represents the largest order of the polynomial we have taken.

Substituting (3) and (2) into (1), we obtain

$$
\begin{aligned}
\left(\sum_{i=0}^{M} x_{i}(n+1) P_{i}(k)\right)= & (1+\bar{\alpha} \beta)\left(\sum_{i=0}^{M} x_{i}(n) P_{i}(k)\right) \\
& +\delta \beta k\left(\sum_{i=0}^{M} x_{i}(n) P_{i}(k)\right) \\
& -\beta\left(\sum_{i=0}^{M} x_{i}(n) P_{i}(k)\right) \\
& \times\left(\sum_{i=0}^{M} y_{i}(n) P_{i}(k)\right), \\
\left(\sum_{i=0}^{M} y_{i}(n+1) P_{i}(k)\right)= & (1-\beta)\left(\sum_{i=0}^{M} y_{i}(n) P_{i}(k)\right) \\
& +\beta\left(\sum_{i=0}^{M} x_{i}(n) P_{i}(k)\right)^{2} .
\end{aligned}
$$

With the help of cycle recurrence formula of Charlier polynomial [25]

$$
k P_{i}^{(\lambda)}(k)=P_{i+1}^{(\lambda)}(k)+(i+\lambda) P_{i}^{(\lambda)}(k)+\lambda i P_{i-1}^{(\lambda)}(k),
$$

the quadratic product polynomial of system (4) can be further reduced into a linear combination of related single polynomials. So the nonlinear terms in system (4) can be written as

$$
\begin{gathered}
\left(\sum_{i=0}^{M} x_{i}(n) P_{i}(k)\right)^{2}=\sum_{i=0}^{M \times 2} S_{i}(n) P_{i}(k), \\
\left(\sum_{i=0}^{M} x_{i}(n) P_{i}(k)\right)\left(\sum_{i=0}^{M} y_{i}(n) P_{i}(k)\right)=\sum_{i=0}^{M \times 2} M_{i}(n) P_{i}(k),
\end{gathered}
$$


where $S_{i}(n)$ and $M_{i}(n)$ are calculated by computer algebraic system. Meantime, the stochastic term in the right hand side of the first equation of system (4) can be simplified as

$$
\begin{aligned}
& \delta \beta k\left(\sum_{i=0}^{M} x_{i}(n) P_{i}(k)\right) \\
& =\delta \beta \sum_{i=0}^{M} x_{i}(n) k P_{i}(k) \\
& =\delta \beta\left(\sum_{i=0}^{M} x_{i}(n)\left(P_{i+1}(k)+(i+\lambda) P_{i}(k)+\lambda i P_{i-1}(k)\right)\right) \\
& =\delta \beta\left[\sum _ { i = 0 } ^ { M } \left(P _ { i } ( k ) \left(\lambda(i+1) x_{i+1}(n)+(i+\lambda) x_{i}(n)\right.\right.\right. \\
& \left.\left.\left.+x_{i-1}(n)\right)-x_{i}(n) P_{i+1}(k)\right)\right],
\end{aligned}
$$

where $x_{-1}$ and $x_{M+1}$ are zero by the principle of approximation.

As $M \rightarrow \infty$, Lorenz discrete system with random parameter is strictly equivalent to system (4) in the sense of mean square convergence. In order to facilitate the numerical analysis in this paper, we select $M=1$. Based on statistical characteristics of the Poisson distribution and the orthogonal polynomial approximation of discrete random function, substituting (6) and (7) into (4) and multiplying $P_{j}(k)(j=0,1)$ in both sides, by taking expectation with respect to $k$, we finally get its approximate equivalent deterministic system of Lorenz discrete system with random parameter as

$$
\begin{aligned}
x_{0}(n+1)= & (1+\bar{\alpha} \beta) x_{0}(n) \\
& +\delta \lambda \beta\left(x_{0}(n)+x_{1}(n)\right)-\beta S_{0}(n), \\
y_{0}(n+1)= & (1-\beta) y_{0}(n)+\beta M_{0}(n), \\
x_{1}(n+1)= & (1+\bar{\alpha} \beta) x_{1}(n)+\delta \beta(1+\lambda) x_{1}(n) \\
& +\delta \beta x_{0}(n)-\beta S_{1}(n), \\
y_{1}(n+1)= & (1-\beta) y_{1}(n)+\beta M_{1}(n) .
\end{aligned}
$$

The stochastic Henon map can be depicted as

$$
\begin{gathered}
x^{*}(n+1)=1-a\left(x^{*}(n)\right)^{2}+y^{*}(n), \\
y^{*}(n+1)=b x^{*}(n),
\end{gathered}
$$

where $a$ is a deterministic parameter, and $b$ is a random parameter. According to the previously mentioned process of
Charlier orthogonal polynomial expansion, the Henon map with random parameter is transformed approximately as

$$
\begin{gathered}
x_{0}^{*}(n+1)=1-a S_{0}^{*}(n)+y_{0}^{*}(n), \\
y_{0}^{*}(n+1)=\bar{b} x_{0}^{*}(n)+\delta \lambda\left(x_{0}^{*}(n)+x_{1}^{*}(n)\right), \\
x_{1}^{*}(n+1)=1-a S_{1}^{*}(n)+y_{1}^{*}(n), \\
y_{1}^{*}(n+1)=\bar{b} x_{1}^{*}(n)+\delta(1+\lambda) x_{1}^{*}(n)+\delta x_{0}^{*}(n) .
\end{gathered}
$$

As parameters of the stochastic Lorenz discrete system are $\bar{\alpha}=1.25, \beta=0.75$, and $\lambda=1$, and the initial condition is chosen as $(0.1,0.2,0.1,0.2)^{T}$, the system (8) with different random intensities shows different chaotic behaviors as shown in Figure 1. For the initial condition $(0.4,0.4,0,0)^{T}$ of stochastic Henon map, parameters are chosen as $a=1.4, \bar{b}=$ 0.3 , and $\lambda=1$; Figure 2 depicts the influence of different random intensities on the stochastic Henon map.

\section{Generalized Synchronization for the Discrete Systems with Random Parameter}

Throughout this paper, $\|\cdot\|_{F}$ denotes the Frobenius norm in the Banach space. The drive and response systems are expressed as

$$
\begin{gathered}
x(n+1)=F(x(n))=A x(n)+f(x(n)), \\
y(n+1)=G(x(n))+u(n)=B y(n)+g(y(n))+u(n),
\end{gathered}
$$

where $x(n), y(n) \in R^{n \times 1}$ are state variables, $F, G, f, g: R^{n} \rightarrow$ $R^{n}, A, B \in R^{n \times n}$, and $u(n)$ is the controller which is used to achieve the generalized synchronization to be designed.

Definition 1. In $n \times n$ matrix $A$, if there exists a constant $\alpha_{A}\left(0 \leq \alpha_{A}<1\right)$ such that the inequality

$$
\|A x-A y\|_{F} \leq \alpha_{A}\|x-y\|_{F}
$$

holds for every pair of points $x, y$, where $x, y$ belong to the Banach space with norm $\|\cdot\|_{F}$, then we say that $A$ is a contraction matrix.

Definition 2. The drive system and the response system are said to be the globally generalized synchronization. If there exists a controller $u(n)$ and a matrix $M$ such that

$$
\lim _{n \rightarrow \infty}\|e(n)\|_{F}=\lim _{n \rightarrow \infty}\|y(n)-M x(n-m)\|_{F}=0,
$$

for any initial conditions. It contains several special cases: if matrix $M$ is a unit matrix and the lag value $m=0(m>$ $0, m<0$ ), the problem can be transformed to complete (lag, anticipated) synchronization; if the lag value $m=n$, it will be converted into chaos control. 


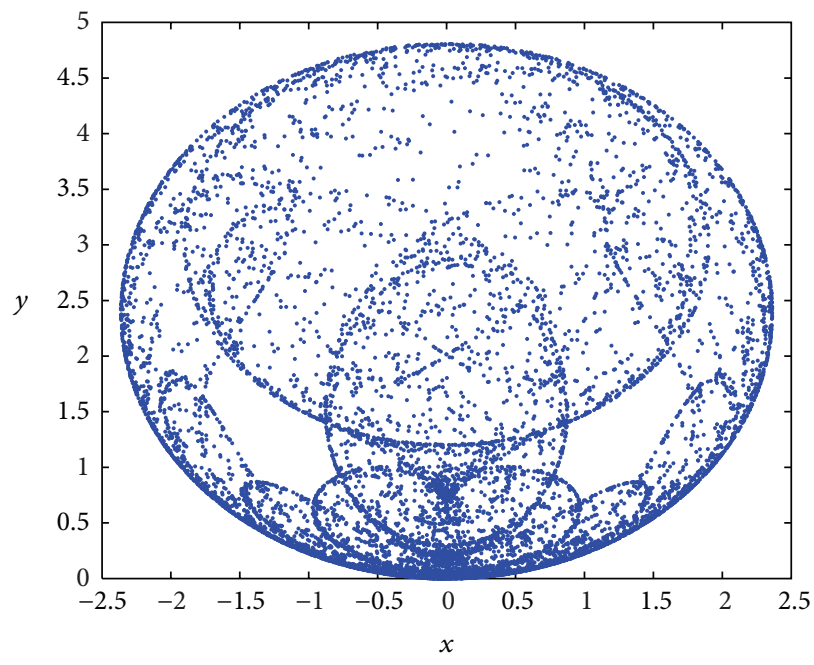

- $\delta=0.00$

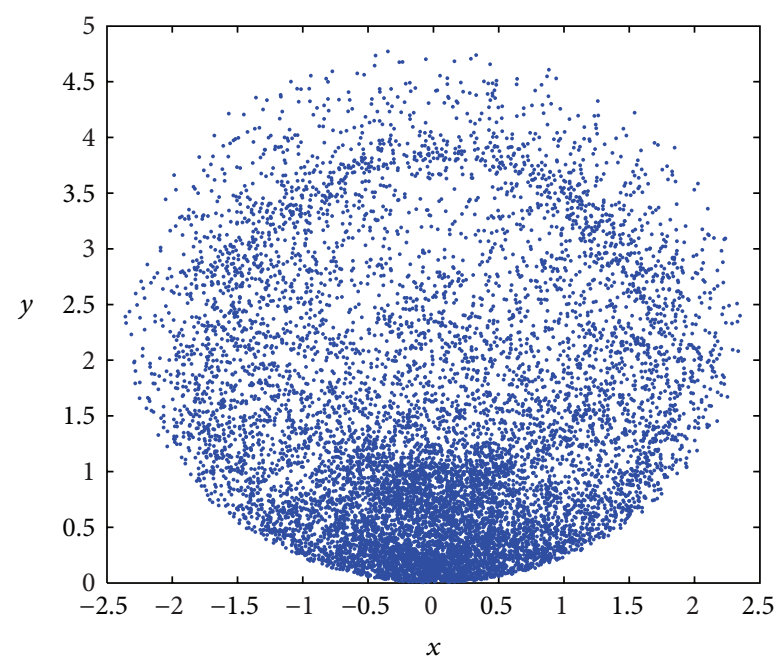

- $\delta=0.003$

(a)

(b)

FIgURE 1: The two attractors of stochastic Lorenz discrete system with $\delta=0.00, \delta=0.003$.

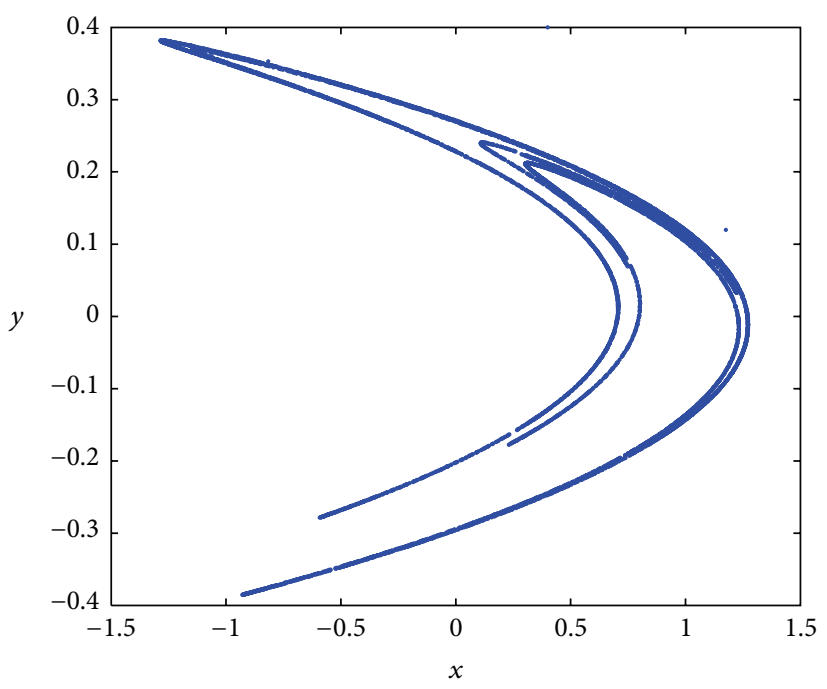

- $\delta=0.00$

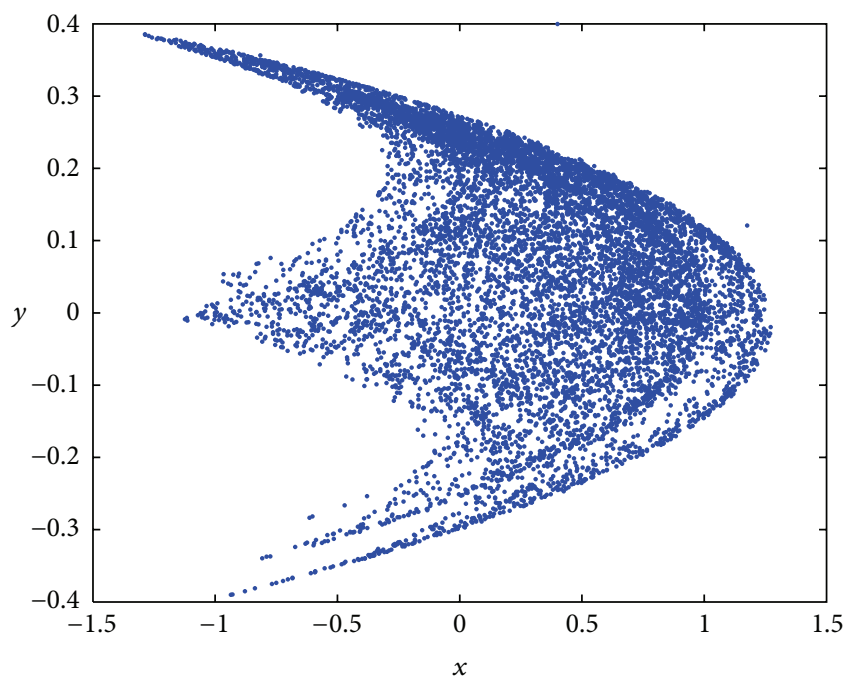

- $\delta=0.002$

(a)

(b)

Figure 2: The two attractors of stochastic Henon map with $\delta=0.00, \delta=0.002$.

Theorem 3. Assume that an $n \times n$ matrix $M$ is exchangeable with matrices $B$ and $C$. If the controller $u(n)$ in response system (12) is given by

$$
\begin{aligned}
u(n)= & M(f(x(n-m))+(A-B+C) x(n-m)) \\
& -g(y(n))-C y(n),
\end{aligned}
$$

where $C \in R^{n \times n}$, then $\lim _{n \rightarrow \infty}\|e(n)\|_{F}=\lim _{n \rightarrow \infty} \| y(n)-$ $M x(n-m) \|_{F}=0$ is satisfied, that is, globally generalized synchronization being achieved under the controller $u(n)$.
Proof. According to the drive system (11) and the response system (12), the error states by Definition 2 can be written as

$$
\begin{aligned}
e(n+1)= & B y(n)+g(y(n))+u(n) \\
& -M(A x(n-m)+f(x(n-m))) \\
= & B y(n)+g(y(n)) \\
& +M(f(x(n-m))+(A-B+C)(x(n-m))) \\
& -g(y(n))-C y(n)
\end{aligned}
$$




$$
\begin{aligned}
& -M(A x(n-m)+f(x(n-m))) \\
= & B y(n)-M B x(n-m)+M C x(n-m)-C y(n) .
\end{aligned}
$$

Because $M B=B M$ and $M C=C M$, then the error system is simplified to $e(n+1)=(B-C) e(n)$. Constructing the Lyapunov function $V_{n}=|e(n)|$, then its derivative is

$$
\Delta V=V_{n+1}-V_{n}=|e(n+1)|-|e(n)|=(|B-C|-1)|e(n)| .
$$

If $\|B-C\| \leq 1$, that is, $B-C$ is a contraction matrix, according to the Lyapunov stability theory and contraction theorem, the error system is globally stable. It means that the generalized synchronization is achieved.

\section{Illustrative Examples}

In this part, to confirm the validity of the proposed control method, we will introduce two types of generalized synchronization for homogeneous and heterogeneous stochastic discrete chaotic systems. In the process of numerical simulation, we choose all random intensity to be $\delta=0.002$.

Example 1. Generalized complete synchronization of the identical stochastic discrete chaotic system.

Consider the stochastic Henon map

$$
\begin{gathered}
x(n+1)=1-a(x(n))^{2}+y(n), \\
y(n+1)=b x(n)
\end{gathered}
$$

as the drive system, in which $b$ is a random parameter and the system (9) is the response system. In order to achieve the generalized synchronization of Henon map with random parameter, we rewrite the system (18) and (9) as

$$
\begin{aligned}
&\left(\begin{array}{l}
x(n+1) \\
y(n+1)
\end{array}\right) \\
&=\left(\begin{array}{ll}
q & p \\
\alpha & \beta
\end{array}\right)\left(\begin{array}{l}
x(n) \\
y(n)
\end{array}\right) \\
&+\left(\begin{array}{r}
1-a(x(n))^{2}+(1-p) y(n)-q x(n) \\
(b-\alpha) x(n)-\beta y(n)
\end{array}\right), \\
&\left(\begin{array}{c}
x^{*}(n+1) \\
y^{*}(n+1)
\end{array}\right) \quad \\
&=\left(\begin{array}{ll}
q & p \\
\alpha & \beta
\end{array}\right)\left(\begin{array}{c}
x^{*}(n) \\
y^{*}(n)
\end{array}\right) \\
& \quad+\left(\begin{array}{c}
1-a\left(x^{*}(n)\right)^{2}+(1-p) y^{*}(n)-q x^{*}(n) \\
(b-\alpha) x^{*}(n)+\beta y^{*}(n)
\end{array}\right) \\
& \quad+u(n), \quad
\end{aligned}
$$

where $|q|,|p|,|\alpha|,|\beta|<1, q, p, \alpha, \beta \neq 0$, and $q, p, \alpha, \beta \in R$. Now, the error state variable is defined as $e(n-1)=(\cdot)^{*}(n)-$ $M(\cdot)(n-m)$. When we select the matrix $C=0, A=B=\left(\begin{array}{cc}q & p \\ \alpha & \beta\end{array}\right)$ is a contraction matrix, and taking the exchangeable matrix $M=\left(\begin{array}{ll}c & d \\ e & f\end{array}\right)(c, d, e, f \in R)$, the generalized synchronization can be realized by Theorem 3 under the following controller:

$$
u(n)=M f(n-m)-f^{*}(n),
$$

where

$$
\begin{gathered}
f(n)=\left(\begin{array}{c}
1-a x^{2}(n)-q x(n)+(1-p) y(n) \\
(b-\alpha) x(n)-\beta y(n)
\end{array}\right), \\
f^{*}(n)=\left(\begin{array}{c}
1-a\left(x^{*}(n)\right)^{2}-q x^{*}(n)+(1-p) y^{*}(n) \\
(b-\alpha) x^{*}(n)-\beta y^{*}(n)
\end{array}\right) .
\end{gathered}
$$

Taking advantage of the Charlier orthogonal polynomial expansion, the stochastic Henon map (19) can be transformed into the following system:

$$
\begin{aligned}
& \left(\begin{array}{l}
x_{0}(n+1) \\
y_{0}(n+1) \\
x_{1}(n+1) \\
y_{1}(n+1)
\end{array}\right) \\
& =\left(\begin{array}{llll}
q & p & 0 & 0 \\
\alpha & \beta & 0 & 0 \\
0 & 0 & q & p \\
0 & 0 & \alpha & \beta
\end{array}\right)\left(\begin{array}{l}
x_{0}(n) \\
y_{0}(n) \\
x_{1}(n) \\
y_{1}(n)
\end{array}\right) \\
& +\left(\begin{array}{rr}
1-S_{0}(n)+(1-p) y_{0}(n)-q x_{0}(n) \\
\\
\quad & (\bar{b}+\delta \lambda-\alpha) x_{0}(n)+\delta \lambda x_{0}(n)-\beta y_{0}(n) \\
\delta x_{0}(n)+(\bar{b}+\delta(\lambda+1)-\alpha) S_{1}(n)-\beta y_{1}(n)+(1-p) y_{1}(n)-q x_{1}(n)
\end{array}\right)
\end{aligned}
$$

Similarly, the system (20) can be reduced to a corresponding equivalent deterministic system. According to the aforementioned conditions, when we choose some appropriate parameter values, the matrix $A=B=\left(\begin{array}{ll}q & p \\ \alpha & \beta\end{array}\right)=$ $\left(\begin{array}{ll}0.1 & 0.2 \\ 0.2 & 0.5\end{array}\right), M=\left(\begin{array}{ll}c & d \\ e & f\end{array}\right)=\left(\begin{array}{cc}1 & 0.5 \\ 4.5 & 0\end{array}\right)$, the standard deviation $\lambda=1$, the lag value $m=0$, and $\lim _{n \rightarrow \infty}\|e(n)\|_{F}=$ $\lim _{n \rightarrow \infty}\left\|(\cdot)^{*}(n)-M(\cdot)(n-m) \cdot\right\|_{F}=0$ are set up. Through the numerical simulation, Figure 3 shows time response diagrams of the generalized complete synchronization error of state variables between the drive system (18) and the response system (9) under the controller $u(n)$ (21) for different initial conditions.

Example 2. Generalized lag synchronization of the different stochastic discrete chaotic system.

Taking the stochastic Lorenz discrete system (1) and the stochastic Henon map (9) as the drive and response systems, we rewrite the drive system (1) and the response system (9) in the following matrices:

$$
\left(\begin{array}{l}
x(n+1) \\
y(n+1)
\end{array}\right)=\left(\begin{array}{cc}
1+\alpha \beta & 0 \\
0 & 1-\beta
\end{array}\right)\left(\begin{array}{l}
x(n) \\
y(n)
\end{array}\right)+\left(\begin{array}{c}
-\beta x(n) y(n) \\
\beta x^{2}(n)
\end{array}\right),
$$

$$
\left(\begin{array}{l}
x^{*}(n+1) \\
y^{*}(n+1)
\end{array}\right)=\left(\begin{array}{ll}
0 & 1 \\
b & 0
\end{array}\right)\left(\begin{array}{l}
x^{*}(n) \\
y^{*}(n)
\end{array}\right)+\left(\begin{array}{c}
1-a\left(x^{*}(n)\right)^{2} \\
0
\end{array}\right)+u(n),
$$



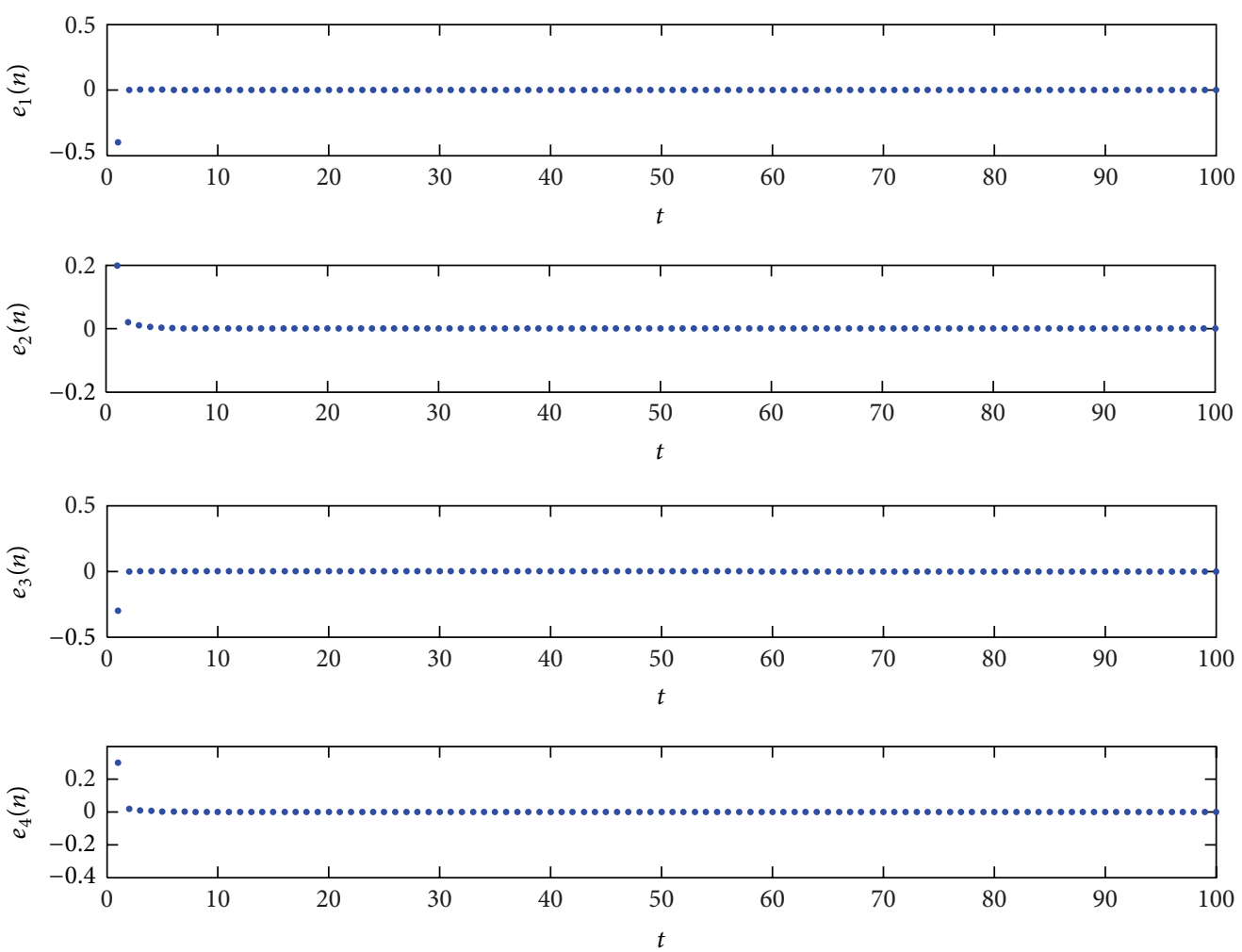

FIGURE 3: The time response of the generalized synchronization error of state variables.

where $\alpha$ and $b$ are random parameters, respectively. By Theorem 3 , on choosing an appropriate matrix $C=\left(\begin{array}{ll}c & d \\ e & f\end{array}\right)$, we design a controller as follows:

$$
\begin{aligned}
u(n)= & M(f(n-m)+(A-B+C) x(n-m)) \\
& -g(y(n))-C y(n),
\end{aligned}
$$

where

$$
\begin{aligned}
& f(n)+(A-B+C) x(n) \\
& \quad=\left(\begin{array}{c}
-\beta x(n) y(n) \\
\beta x^{2}(n)
\end{array}\right)+\left(\begin{array}{cc}
1+\alpha \beta+c & -1+d \\
-b+e & 1-\beta+f
\end{array}\right)\left(\begin{array}{l}
x(n) \\
y(n)
\end{array}\right), \\
& g(y(n))+C y(n)=\left(\begin{array}{c}
1-a\left(x^{*}(n)\right)^{2} \\
0
\end{array}\right)+\left(\begin{array}{ll}
c & d \\
e & f
\end{array}\right)\left(\begin{array}{l}
x^{*}(n) \\
y^{*}(n)
\end{array}\right) .
\end{aligned}
$$

By applying the Charlier orthogonal polynomial expansion, the controlled response system (25) with random parameter can be reduced into an equivalent deterministic controlled system. The matrices $A, B$ and $C$ can be converted into the corresponding fourth-order square matrix. So, its corresponding fourth-order matrix of $B$ is

$$
B^{\prime}=\left(\begin{array}{cccc}
0 & 1 & 0 & 0 \\
\bar{b}+\delta \lambda & 0 & \delta \lambda & 0 \\
0 & 0 & 0 & 1 \\
\delta & 0 & \bar{b}+\delta(1+\lambda) & 0
\end{array}\right)
$$

For the unit matrix being exchangeable with any square matrix, when we choose the matrix $M=q I(q \in N)$, then the error state equation $e(n+1)=\left(B^{\prime}-C^{\prime}\right) e(n)$ is stable if we choose an appropriate matrix $C$ such that the corresponding matrix $C^{\prime}$ of $C$ satisfies that $B^{\prime}-C^{\prime}$ is a contraction matrix. With the given initial condition, the lag value $m=5, q=$ $3, \lambda=1$, and $\bar{b}=0.3$, we have

$$
C^{\prime}=\left(\begin{array}{cccc}
-0.5 & 0.5 & 0 & 0 \\
0 & 0.2 & 0 & 0 \\
0 & 0 & -0.5 & 0.5 \\
0 & 0 & 0 & 0.2
\end{array}\right)
$$

that is, $c=-d=0.5, e=0$, and $f=0.2$; the time response of the generalized lag synchronization error of state variables is shown in Figure 4.

\section{Conclusions}

The problem of generalized synchronization for stochastic discrete chaotic system by active control is investigated in this paper. By the approximation principle of orthogonal polynomial, we transformed the stochastic discrete chaotic system with Poisson distribution coefficient into the equivalent deterministic discrete system. According to Lyapunov stability theory and contraction matrix theorem, an active controller has been proposed for the generalized synchronization of those stochastic discrete systems. The illustrative results show that this active controller can be used to achieve 

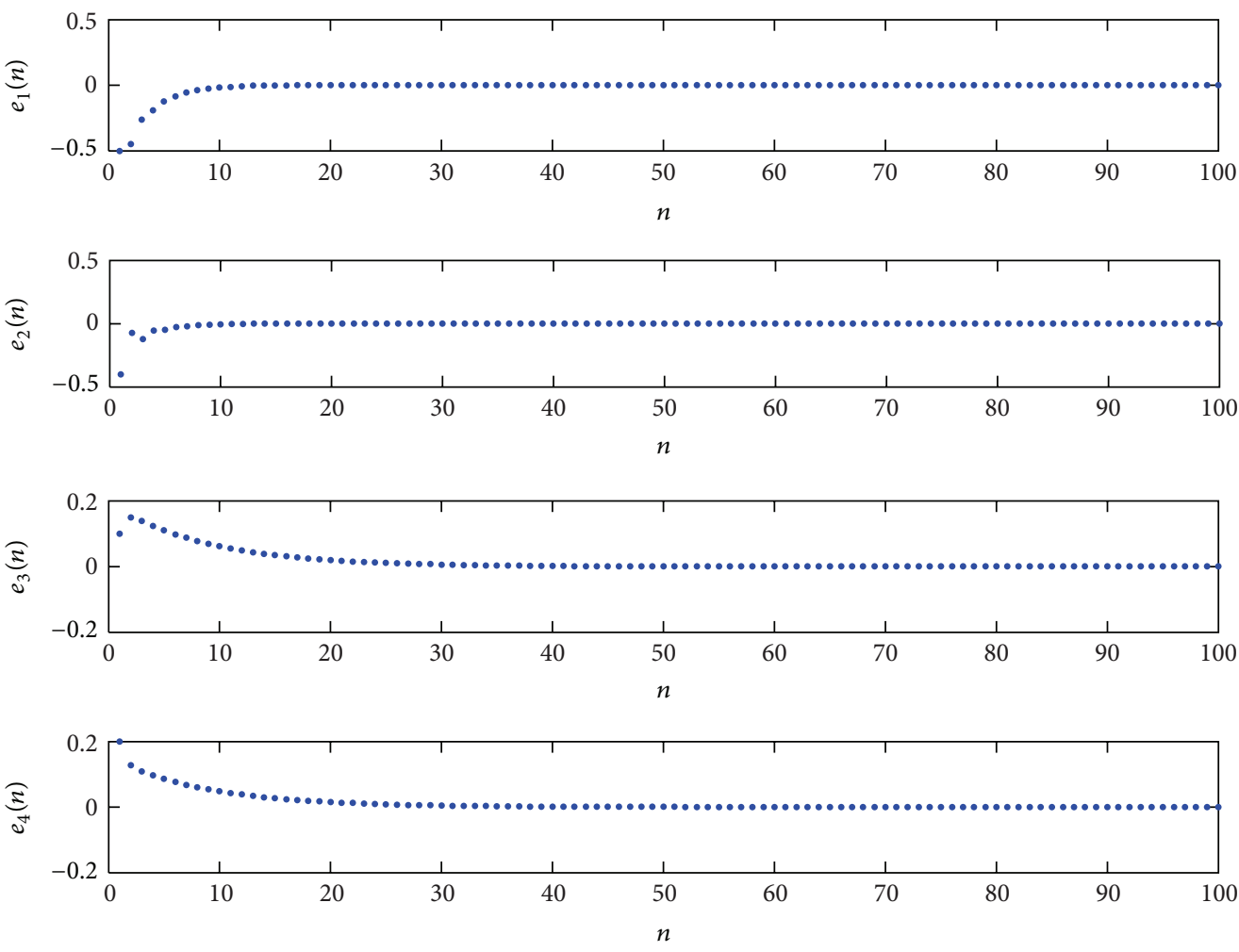

FIGURE 4: The time response of the synchronization error of state variables.

the synchronization of any stochastic discrete system with random parameter.

\section{Appendix}

$S_{i}(n), M_{i}(n)(i=0,1)$ in $(8)$ can be derived through the computer algebraic system, such as Maple, as follows:

$$
\begin{gathered}
S_{0}(n)=x_{0}^{2}(n)+2 x_{1}^{2}(n), \\
S_{1}(n)=2 x_{0}(n) x_{1}(n)+2 x_{1}^{2}(n), \\
M_{0}(n)=x_{0}(n) y_{0}(n)+2 x_{1}(n) y_{1}(n), \\
M_{1}(n)=x_{0}(n) y_{1}(n)+x_{1}(n) y_{0}(n)+2 x_{1}(n) y_{1}(n) .
\end{gathered}
$$

$S_{i}^{*}(n)(i=0,1)$ in $(10)$ can be calculated by Maple as follows:

$$
\begin{gathered}
S_{0}^{*}(n)=\left(x_{0}^{*}(n)\right)^{2}+2\left(x_{1}^{*}(n)\right)^{2}, \\
S_{1}^{*}(n)=2 x_{0}^{*}(n) x_{1}^{*}(n)+2\left(x_{1}^{*}(n)\right)^{2} .
\end{gathered}
$$

\section{Acknowledgments}

This project is supported by the National Natural Science Foundation of China (Grant no. 11002001), the Natural Science Foundation of Ningxia Hui Autonomous Region (Grant no. NZ12210), and the innovative funded project for the postgraduate students of Beifang University of Nationalities. The authors thank all of the referees for their valuable comments and suggestions leading to the improvement of this paper.

\section{References}

[1] L. M. Pecora and T. L. Carroll, "Synchronization in chaotic systems," Physical Review Letters, vol. 64, no. 8, pp. 821-824, 1990.

[2] G. Chen and X. Dong, From Chaos to Order Methodologies, Perspectives and Applications, with a Foreword by Alistair Mees, vol. 24 of World Scientific Series on Nonlinear Science. Series A: Monographs and Treatises, World Scientific Publishing, River Edge, NJ, USA, 1998.

[3] H. Taghvafard and G. H. Erjaee, "Phase and anti-phase synchronization of fractional order chaotic systems via active control," Communications in Nonlinear Science and Numerical Simulation, vol. 16, no. 10, pp. 4079-4088, 2011.

[4] D. Yu and U. Parlitz, "Partial synchronization of chaotic systems with uncertainty," Physical Review E, vol. 77, no. 6, Article ID 066208, 2008.

[5] B. Xin, T. Chen, and Y. Liu, "Projective synchronization of chaotic fractional-order energy resources demand-supply systems via linear control," Communications in Nonlinear Science and Numerical Simulation, vol. 16, no. 11, pp. 4479-4486, 2011.

[6] H. Du, P. Shi, and N. Lü, "Function projective synchronization in complex dynamical networks with time delay via hybrid feedback control," Nonlinear Analysis: Real World Applications, vol. 14, no. 2, pp. 1182-1190, 2013. 
[7] H.-G. Zhang, T.-D. Ma, J. Fu, and S.-C. Tong, "Robust lag synchronization between two different chaotic systems via dualstage impulsive control," Chinese Physics B, vol. 18, no. 9, pp. 3751-3757, 2009.

[8] Y. Chenggui, Z. Qi, and Y. Jun, "Complete synchronization induced by disorder in coupled chaotic lattices," Physics Letters A, vol. 377, no. 5, pp. 370-377, 2013.

[9] J. Zhao and T. Ren, "Q-S synchronization between chaotic systems with double scaling functions," Nonlinear Dynamics, vol. 62, no. 3, pp. 665-672, 2010.

[10] S. Cheng, J. C. Ji, and J. Zhou, "Fast synchronization of directionally coupled chaotic systems," Applied Mathematical Modelling, vol. 37, no. 1-2, pp. 127-136, 2013.

[11] X. Wan and J. Sun, "Adaptive-impulsive synchronization of chaotic systems," Mathematics and Computers in Simulation, vol. 81, no. 8, pp. 1609-1617, 2011.

[12] M. Peng, E.-W. Bai, and K. E. Lonngren, "On the synchronization of delay discrete models," Chaos, Solitons and Fractals, vol. 22, no. 3, pp. 573-576, 2004.

[13] G. Baier and M. Klein, "Maximum hyperchaos in generalized Hénon maps," Physics Letters A, vol. 151, no. 6-7, pp. 281-284, 1990.

[14] B. Chen and J. Chen, "Bifurcation and chaotic behavior of a discrete singular biological economic system," Applied Mathematics and Computation, vol. 219, no. 5, pp. 2371-2386, 2012.

[15] Z. Zhang and X. Liu, "Observer-based impulsive chaotic synchronization of discrete-time switched systems," Nonlinear Dynamics, vol. 62, no. 4, pp. 781-789, 2010.

[16] Z.-Y. Wu and X.-C. Fu, "Adaptive function projective synchronization of discrete chaotic systems with unknown parameters," Chinese Physics Letters, vol. 27, no. 5, Article ID 050502, 2010.

[17] K. Hengster-Movric, K. You, F. L. Lewis, and L. Xie, "Synchronization of discrete-time multi-agent systems on graphs using Riccati design," Automatica, vol. 49, no. 2, pp. 414-423, 2013.

[18] W. Liu, Z. Wang, and W. Zhang, "Controlled synchronization of discrete-time chaotic systems under communication constraints," Nonlinear Dynamics, vol. 69, no. 1-2, pp. 223-230, 2012.

[19] N. Vasegh and V. J. Majd, "Adaptive fuzzy synchronization of discrete-time chaotic systems," Chaos, Solitons and Fractals, vol. 28, no. 4, pp. 1029-1036, 2006.

[20] H. Su and X. H. Ding, "Synchronization in time-discrete delayed chaotic systems," Neurocomputing, vol. 73, no. 1-3, pp. 478-483, 2009.

[21] Z. Yan, "Q-S (complete or anticipated) synchronization backstepping scheme in a class of discrete-time chaotic (hyperchaotic) systems: a symbolic-numeric computation approach," Chaos, vol. 16, no. 1, Article ID 013119, 2006.

[22] H.-L. An and Y. Chen, "The function cascade synchronization scheme for discrete-time hyperchaotic systems," Communications in Nonlinear Science and Numerical Simulation, vol. 14, no. 4, pp. 1494-1501, 2009.

[23] M. Eisencraft and A. M. Batista, "Discrete-time chaotic systems synchronization performance under additive noise," Signal Processing, vol. 91, no. 8, pp. 2127-2131, 2011.

[24] G. Grassi and D. A. Miller, "Dead-beat full state hybrid projective synchronization for chaotic maps using a scalar synchronizing signal," Communications in Nonlinear Science and Numerical Simulation, vol. 17, no. 4, pp. 1824-1830, 2012.

[25] P. Borwein and T. Erdélyi, Polynomials and Polynomial Inequality, Springer, New York, NY, USA, 1995. 


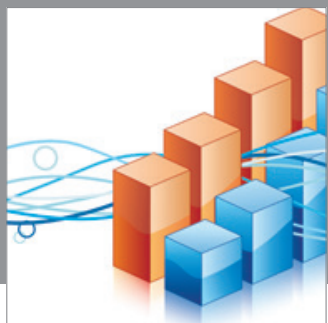

Advances in

Operations Research

mansans

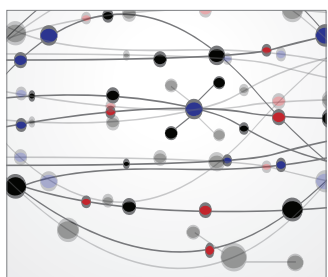

The Scientific World Journal
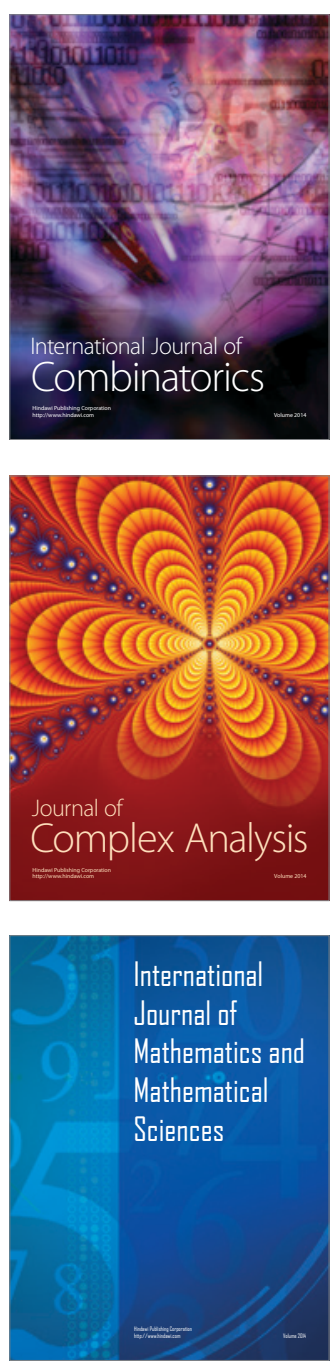
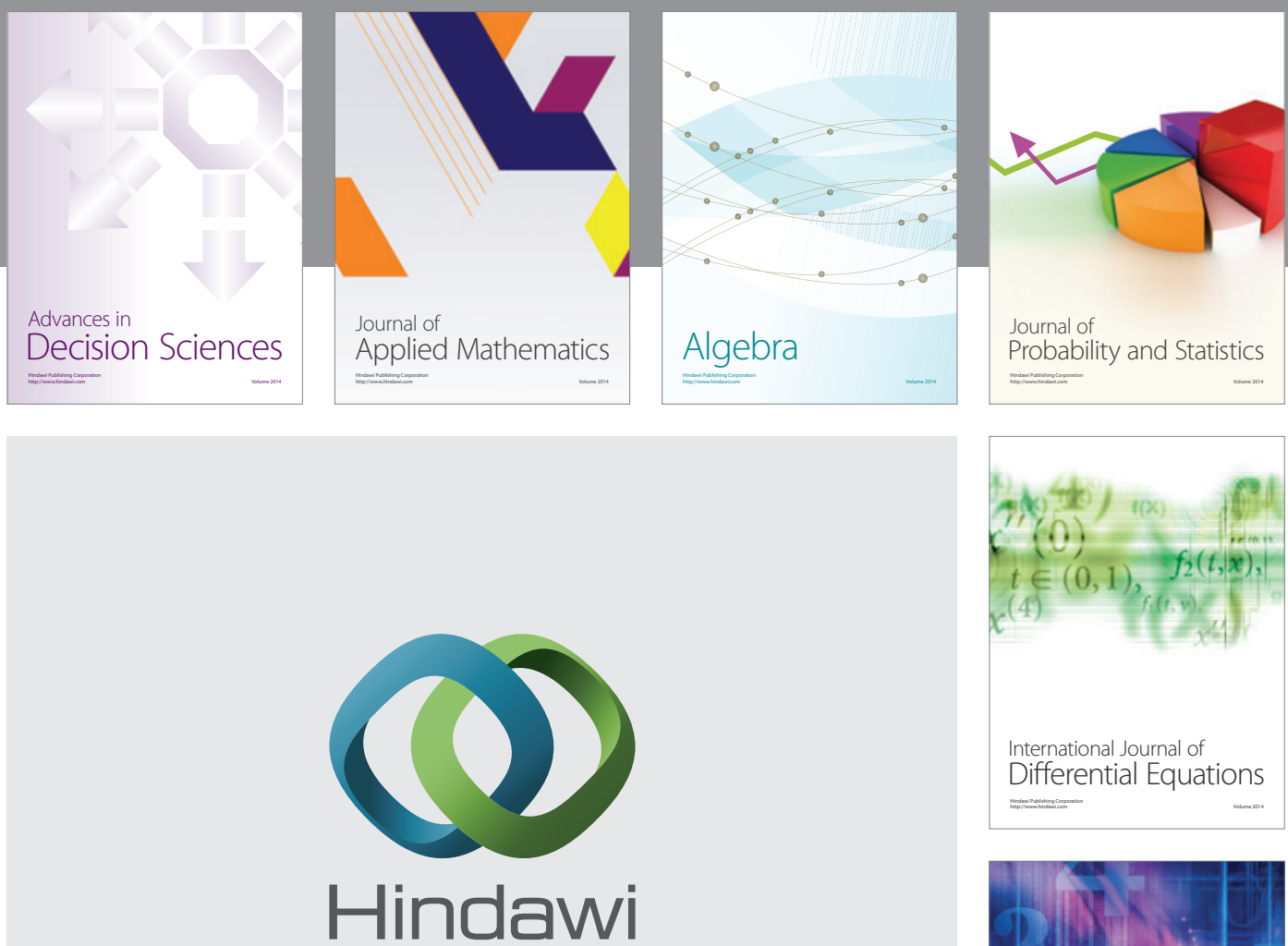

Submit your manuscripts at http://www.hindawi.com
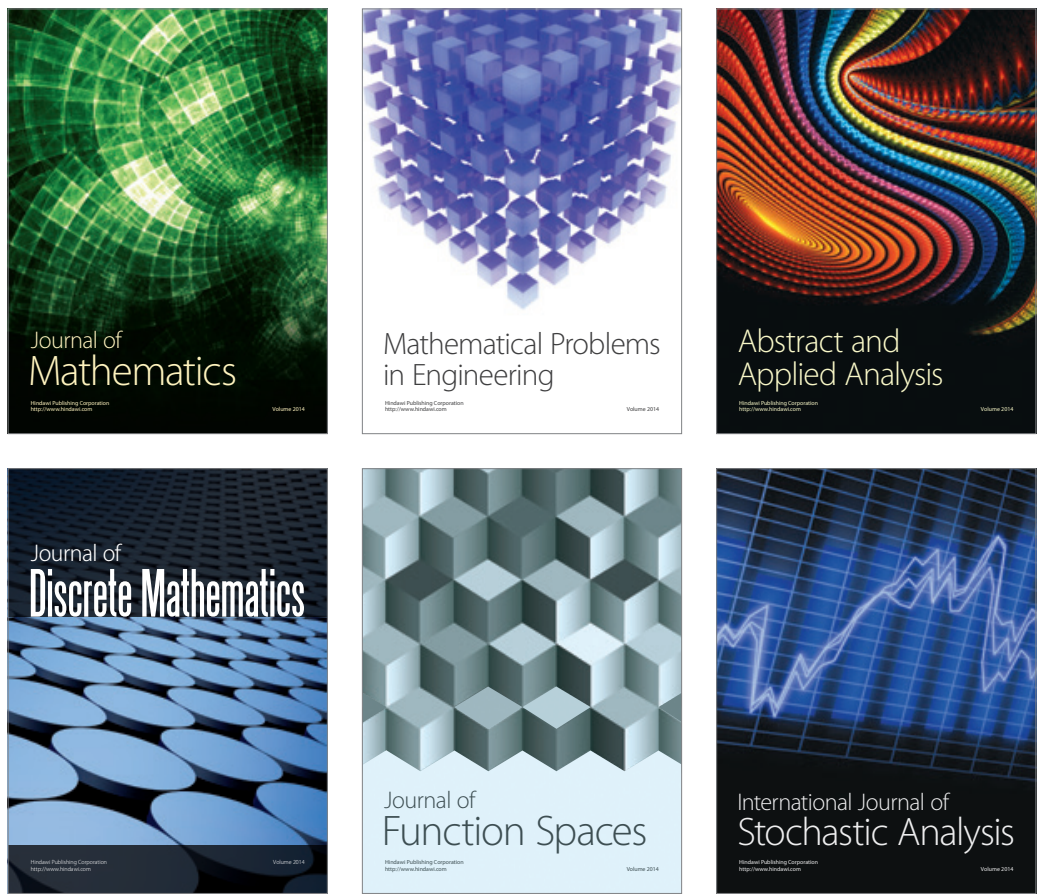

Journal of

Function Spaces

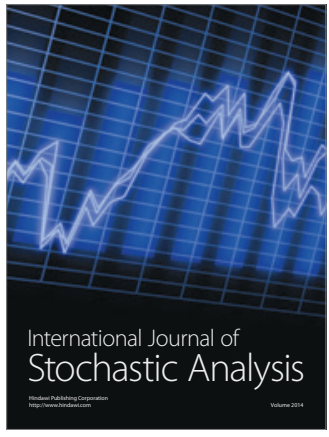

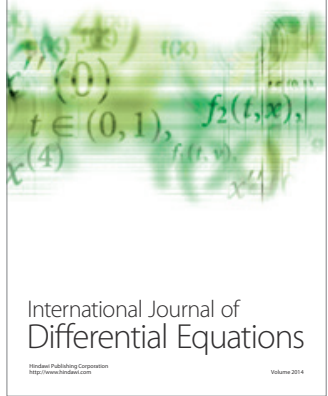
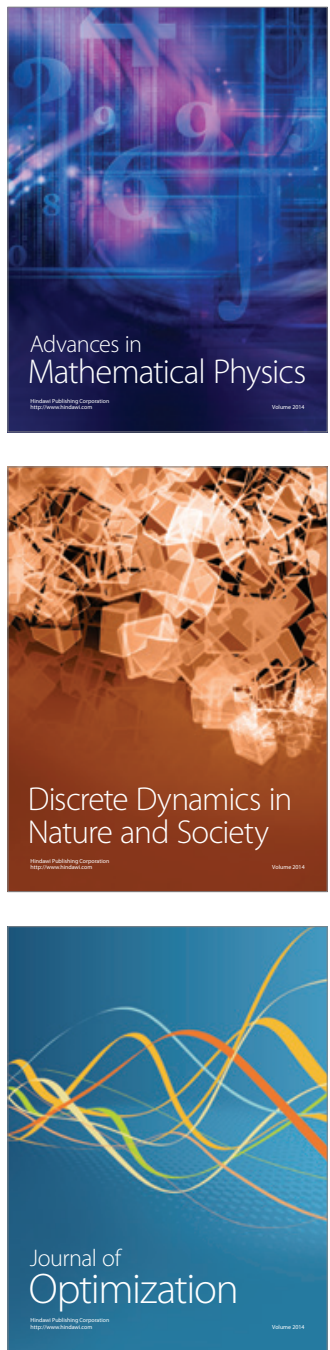